\title{
ON THE SOLVABILITY OF HIGHER-ORDER OPERATOR-DIFFERENTIAL EQUATIONS IN A WEIGHTED SOBOLEV SPACE
}

\author{
Abdel Baset I. Ahmed \\ Eng. Maths. and Physics Dept., Helwan University \\ Cairo - 11865, EGYPT
}

\begin{abstract}
In a weighted Sobolev space on the whole real axis, we obtain the sufficient conditions for the well-posed and unique solvability of $m, n$ order operator-differential equations. These conditions were formulated only by the operator coefficients of the considered equation. According to the values of $m, n$ the operator-differential equation has complicated and multiple characteristics. In addition, by using the main part of the equation, the norms of the operators of intermediate derivative were estimated. We deduce the relationship between the exponent of the weight and the lower bound of the spectrum of the operator of the main part of the equation. As an applied result of this paper, we formulated a problem for higher-order partial differential equations and we provided an alternative method for obtaining the regular solvability of operator pencil.
\end{abstract}

AMS Subject Classification: 47D03, 34A12, 34G10

Key Words: regular solvability; operator-differential equation; self-adjoint operator; weighted Sobolev space; operator pencil

\section{Introduction}

The theory of operator-differential equations in Banach or Hilbert space played a key role in searching both of the ordinary and the partial differential operators (see [25]). In this paper, the investigated equation expressed an interest in applications, for instance, the dynamic problems of arches as well as rings and

Received: September 25, 2020

(C) 2021 Academic Publications 
modeling the stability of the plates from plastic (see [21], [25]). The solvability of initial boundary value problems for higher order operator differential equations has been researched by many authors as A.A. Gasymov, V.I. Gorbachuk, M.L. Gorbachuk, S.Ya. Yakubov, V.N. Pilipchuk and their followers (see [6], [9], [19], [20], [22], [24]). Nowadays, a large number of papers concerning the study of solvability of the operator differential equations in Hilbert or Banach spaces has been published. The principal part of the investigated equation has mixed multiple-complicated characteristics with $m, n$ order $(m, n \geq 1)$. It should be noted that for specific values of $m, n$, the solvability problem for second $(m=0, n=2)$, third $(m=1, n=2)$, fourth $(m=1, n=3)$ and fifth $(m=5, n=0)$ - order operator differential equations have been studied elsewhere (see [1]-[7], [16]). This study differs from the study in work [5]. The main part of the equation in work [5] contains $\frac{d u(t)}{d t}$ and $\frac{d^{n} u(t)}{d t^{n}}$ terms, $(t \in[0,+\infty))$ with only multiple characteristics while in this study the solvability problem for operator-differential equations include $\frac{d^{m} u(t)}{d t^{m}}$ and $\frac{d^{n} u(t)}{d t^{n}}$ terms, $(t \in(-\infty,+\infty))$ with both complicated and multiple characteristics. The $m, n$-order differential equations are very difficult to solve because they must be solved in more complete form as the main part of the equation contains $\frac{d^{m} u(t)}{d t^{m}}$ and $\frac{d^{n} u(t)}{d t^{n}}$ terms. In the whole real axis and in a weighted Sobolev space, the general higherorder operator-differential equations with complicated and multiple characteristic have not been studied yet. The interest of this paper is to provide a general case of the solvability for operator-differential equations in a weighted Sobolev space that cover many applications in the future.

In a separable Hilbert space $H$, we have the following operator-differential equations:

$$
\begin{gathered}
\left(\frac{d}{d t}-A\right)^{m}\left(\frac{d}{d t}+A\right)^{n} u(t)+\sum_{j=0}^{m+n} A_{m+n-j} u^{(j)}(t)=f(t) \\
t \in R=(-\infty,+\infty),
\end{gathered}
$$

where $A$ is a self-adjoint positive-definite operator $\left(A=A^{*} \geq \sigma_{0} E, \sigma_{0}>0\right)$, $\sigma_{0}$ is the lower bound of spectrum $\left(\sigma_{0} \in \sigma(A)\right), E$ is the unit operator, and $A_{j}, j=\overline{0, m+n}$ are generally linear unbounded operators. All derivatives are understood in the sense of distributions theory. We consider $f(t) \in L_{2, \alpha}=$ 
$L_{2, \alpha}(R ; H), u(t) \in W_{2, \alpha}^{n+m}(R ; H)$, and $\alpha \in R$, where

$$
\begin{gathered}
L_{2, \alpha}=\left\{f(t):\|f(t)\|_{L_{2, \alpha}}=\left(\int_{-\infty}^{+\infty}\|f(t)\|_{H}^{2} e^{-\alpha t} d t\right)^{\frac{1}{2}}<+\infty\right\}, \\
W_{2, \alpha}^{n+m}(R ; H)=\left\{u(t): \frac{d^{n+m} u(t)}{d t^{n+m}} \in L_{2, \alpha}, A^{n+m} u(t) \in L_{2, \alpha}\right\}, \\
\|u\|_{W_{2, \alpha}^{n+m}(R ; H)} \\
=\left(\int_{-\infty}^{+\infty}\left(\left\|A^{n+m}(u)\right\|_{L_{2, \alpha}}^{2}+\left\|\frac{d^{n+m}(u)}{d t^{n+m}}\right\|_{L_{2, \alpha}}^{2} e^{-\alpha t} d t\right)\right)^{\frac{1}{2}}<+\infty .
\end{gathered}
$$

At $\alpha=0$, for simplification we denote the space $L_{2,0}(R ; H)$ by $L_{2}(R ; H)$ and the space $W_{2,0}^{n+m}(R ; H)$ by $W_{2}^{n+m}(R ; H)$ (see [15], [18]).

Definition 1. If for any $f(t) \in L_{2, \alpha}(R ; H)$ there exists a vector function $u(t) \in W_{2, \alpha}^{n+m}(R ; H)$ that satisfies (1) almost everywhere in $R$, and the inequality

$$
\|u\|_{W_{2, \alpha}^{n+m}(R ; H)} \leq \text { const }\|f\|_{L_{2, \alpha}}
$$

is true, then $u(t)$ is called a regular solution of equation (1) and equation (1) is called regularly solvable (see [1], [2], [12], [16]).

We denote

$$
\begin{gathered}
P_{0} u(t)=\left(\frac{d}{d t}-A\right)^{m}\left(\frac{d}{d t}+A\right)^{n} u(t), \\
P_{1} u(t)=\sum_{j=0}^{m+n} A_{m+n-j} u^{(j)}(t) .
\end{gathered}
$$

Then equation (1) can be written in the form

$$
P u(t) \equiv P_{0} u(t)+P_{1} u(t)=f(t), t \in R .
$$

\section{Main results}

Theorem 2. Let $|\alpha|<2 \sigma_{0}$. Then the operator $P_{0}$ is an isomorphism from $W_{2, \alpha}^{m+n}(R ; H)$ to $L_{2, \alpha}(R ; H)$ (see [2]-[6], [11]). 
Proof. Equation (2) can be written in the form:

$$
P_{0}\left(\frac{d}{d t} ; A\right) u(t)=f(t)
$$

where $f(t) \in L_{2, \alpha}(R ; H), u(t) \in W_{2, \alpha}^{n+m}(R ; H)$.

Let $u(t)=v(t) e^{\frac{\alpha}{2} t}$, then equation (2) takes the form

$$
P_{0, \alpha}\left(\frac{d}{d t}+\frac{\alpha}{2} ; A\right) v(t)=g(t)
$$

where $v(t) \in W_{2}^{n+m}(R ; H), g(t)=f(t) e^{\frac{-\alpha}{2} t} \in L_{2}(R ; H)$. Since the mapping $v(t) \rightarrow u(t) e^{-\frac{\alpha}{2} t}$ is an isomorphism between the spaces $W_{2}^{n+m}(R ; H)$ and $W_{2, \alpha}^{n+m}(R ; H)$, It is sufficient to prove that $P_{0, \alpha}: W_{2}^{m+n}(R ; H) \rightarrow L_{2}(R ; H)$ is an isomorphism, where

$$
P_{0, \alpha} v(t) \equiv\left(\frac{d}{d t}+\frac{\alpha}{2}-A\right)^{m}\left(\frac{d}{d t}+\frac{\alpha}{2}+A\right)^{n} v(t)=g(t) .
$$

So we must find the solution of (6) in the form

$$
v(t)=\int_{-\infty}^{+\infty} G(t-s) g(s) d s \equiv P_{0, \alpha}^{-1} g .
$$

By using Fourier transform for (6), we obtain

$$
\left(i \zeta E+\frac{\alpha}{2} E-A\right)^{m}\left(i \zeta E+\frac{\alpha}{2} E+A\right)^{n} \tilde{v}(\zeta)=\tilde{g}(\zeta), \quad \zeta \in R,
$$

where $\tilde{v}(\zeta)$ and $\tilde{g}(\zeta)$ are Fourier transforms for the functions $v(t)$ and $g(t)$, respectively. For $|\alpha|<2 \sigma_{0}$, the operator pencil

$$
\left(i \zeta E+\frac{\alpha}{2} E-A\right)^{m}\left(i \zeta E+\frac{\alpha}{2} E+A\right)^{n} \tilde{v}(\zeta)=\tilde{g}(\zeta)
$$

is invertible and moreover,

$$
\tilde{v}(\zeta)=\left(i \zeta E+\frac{\alpha}{2} E-A\right)^{-m}\left(i \zeta E+\frac{\alpha}{2} E+A\right)^{-n} \tilde{g}(\zeta)
$$

hence 


$$
\begin{aligned}
& v(t)=\frac{1}{\sqrt{2 \pi}} \int_{-\infty}^{+\infty}\left(i \zeta E+\frac{\alpha}{2} E-A\right)^{-m}\left(i \zeta E+\frac{\alpha}{2} E+A\right)^{-n} \\
& \times \tilde{g}(\zeta) e^{i \zeta t} d \zeta, \quad t \in R \\
& =\frac{1}{2 \pi} \int_{-\infty}^{+\infty}\left(i \zeta E+\frac{\alpha}{2} E-A\right)^{-m}\left(i \zeta E+\frac{\alpha}{2} E+A\right)^{-n} \\
& \times\left(\int_{-\infty}^{+\infty} g(s) e^{-i \zeta s} d s\right) e^{i \zeta t} d \zeta \\
& =\int_{-\infty}^{+\infty} \frac{1}{2 \pi} \int_{0}^{+\infty}\left(i \zeta E+\frac{\alpha}{2} E-A\right)^{-m}\left(i \zeta E+\frac{\alpha}{2} E+A\right)^{-n} e^{i \zeta(t-s)} \\
& \times d \zeta g(s) d s=\int_{-\infty}^{+\infty} G(t-s) g(s) d s,
\end{aligned}
$$

then

$$
G(t-s)=\frac{1}{2 \pi} \int_{-\infty}^{+\infty}\left(i \zeta E+\frac{\alpha}{2} E-A\right)^{-m}\left(i \zeta E+\frac{\alpha}{2} E+A\right)^{-n} e^{i \zeta(t-s)} d \zeta
$$

By taking $i \zeta=w$, then

$$
G(t-s)=\frac{1}{2 \pi i} \int_{-i \infty}^{+i \infty}\left(\omega E+\frac{\alpha}{2} E-A\right)^{-m}\left(\omega E+\frac{\alpha}{2} E+A\right)^{-n} e^{w(t-s)} d \omega
$$

If $\mu \in \sigma(A)$, then

$$
G(t-s)=\frac{1}{2 \pi i} \int_{-i \infty}^{+i \infty} \frac{e^{\omega(t-s)}}{\left(\omega+\frac{\alpha}{2}-\mu\right)^{m}\left(\omega+\frac{\alpha}{2}+\mu\right)^{n}} d \omega
$$

Using the Cauchy integral:

If $t>s$, we get 


$$
\begin{aligned}
& G(t-s)=\operatorname{Re~}_{\omega=-\mu-\frac{\alpha}{2}} \frac{e^{\omega(t-s)}}{\left(\omega+\frac{\alpha}{2}-\mu\right)^{m}\left(\omega+\frac{\alpha}{2}+\mu\right)^{n}} \\
& =\frac{1}{(n-1) !} \lim _{\omega \rightarrow-\mu-\frac{\alpha}{2}} \frac{d^{n-1}}{d w^{n-1}}\left[\frac{e^{\omega(t-s)}}{\left(\omega+\frac{\alpha}{2}-\mu\right)^{m}}\right] \\
& =\frac{1}{(n-1) !} \sum_{k=0}^{2} \frac{2^{-(n+m)+1}(n-k)(n-1) ![\mu(t-s)]^{k}}{2^{(-k)} k ! \mu^{(n+m)-1}} e^{-\left(\mu+\frac{\alpha}{2}\right)(t-s)} \\
& \left.+\frac{1}{(n-1) !} \sum_{k=3}^{n-1} \frac{2^{-(n+m)+1}(n-1) ![\mu(t-s)]^{k}}{2^{(-k)} k ! \mu} e^{-(n+m)-1} \frac{\alpha}{2}\right)(t-s) \\
& =\sum_{k=0}^{2} \frac{2^{-(n+m)+1}(n-k)[\mu(t-s)]^{k}}{2^{(-k)} k !} e^{-\left(\mu+\frac{\alpha}{2}\right)(t-s)} \mu^{-(n+m)+1} \\
& +\sum_{k=3}^{n-1} \frac{2^{-(n+m)+1}[\mu(t-s)]^{k}}{2^{(-k)} k !} e^{-\left(\mu+\frac{\alpha}{2}\right)(t-s)} \mu^{-(n+m)+1}
\end{aligned}
$$

similarly for $t<s$, we have

$$
\begin{aligned}
& G(t-s)=\operatorname{Re} s_{\omega=\mu-\frac{\alpha}{2}} \frac{e^{\omega(t-s)}}{\left(\omega+\frac{\alpha}{2}-\mu\right)^{m}\left(\omega+\frac{\alpha}{2}+\mu\right)^{n}} \\
& =\frac{1}{(m-1) !} \lim _{\omega \rightarrow \mu-\frac{\alpha}{2}} \frac{d^{m-1}}{d w^{m-1}}\left[\frac{e^{\omega(t-s)}}{\left(\omega+\frac{\alpha}{2}+\mu\right)^{n}}\right] \\
& =\frac{1}{(m-1) !} \sum_{k=0}^{2} \frac{2^{-(n+m)+1}(m-k)(m-1) ![\mu(t-s)]^{k}}{2^{(-k)} k ! \mu^{(n+m)-1}} e^{\left(\mu-\frac{\alpha}{2}\right)(t-s)} \\
& +\frac{1}{(m-1) !} \sum_{k=3}^{m-1} \frac{2^{-(n+m)+1}(m-1) ![\mu(t-s)]^{k}}{2^{(-k)} k ! \mu(n+m)-1} e^{\left(\mu-\frac{\alpha}{2}\right)(t-s)} \\
& =\sum_{k=0}^{2} \frac{2^{-(n+m)+1}(m-k)[\mu(t-s)]^{k}}{2^{(-k)} k !} e^{\left(\mu-\frac{\alpha}{2}\right)(t-s)} \mu^{-(n+m)+1} \\
& +\sum_{k=3}^{m-1} \frac{2^{-(n+m)+1}[\mu(t-s)]^{k}}{2^{(-k)} k !} e^{\left(\mu-\frac{\alpha}{2}\right)(t-s)} \mu^{-(n+m)+1} .
\end{aligned}
$$


From the spectral expansion of operator $A,(\mu \in \sigma(A)$, we get

$$
G(t-s)=\left\{\begin{array}{l}
\sum_{k=0}^{2} Q_{m, n}[A(t-s)]^{k} e^{-\left(A+\frac{\alpha}{2} E\right)(t-s)} A^{-(n+m)+1} \\
+\sum_{k=3}^{n-1} R_{m, n}[A(t-s)]^{k} e^{-\left(A+\frac{\alpha}{2} E\right)(t-s)} A^{-(n+m)+1}, t>s \\
\sum_{k=0}^{2} S_{m, n}\left[\left(A-\frac{\alpha}{2} E\right)(t-s)\right]^{k} e^{\left(A-\frac{\alpha}{2} E\right)(t-s)} A^{-(n+m)+1} \\
+\sum_{k=3}^{m-1} T_{m, n}[A(t-s)]^{k} e^{\left(A-\frac{\alpha}{2} E\right)(t-s)} A^{-(n+m)+1}, t<s,
\end{array}\right.
$$

where

$$
\begin{gathered}
Q_{m, n}=\frac{2^{-(n+m)+1}(n-k)}{2^{(-k)} k !}, \quad R_{m, n}=\frac{2^{-(n+m)+1}}{2^{(-k)} k !}, \\
S_{m, n} \frac{2^{-(n+m)+1}(m-k)}{2^{(-k)} k !}, \quad T_{m, n} \frac{2^{-(n+m)+1}}{2^{(-k)} k !}, \quad m, n \geq 2 .
\end{gathered}
$$

The solution $v(t)$ satisfies equation (6) almost everywhere.

According to (8), now we show that $v(t) \in W_{2}^{m+n}(R ; H)$. By using Parseval's equality, we obtain:

$$
\begin{aligned}
& \|v\|_{W_{2}^{m+n}(R ; H)}^{2}=\left\|\frac{d^{m+n} v}{d t^{m+n}}\right\|_{L_{2}(R ; H)}^{2}+\left\|A^{m+n} v\right\|_{L_{2}(R ; H)}^{2} \\
& =\left\|(i \zeta)^{m+n} \tilde{v}(\zeta)\right\|_{L_{2}(R ; H)}^{2}+\left\|A^{m+n} \tilde{v}(\zeta)\right\|_{L_{2}(R ; H)}^{2} \\
& =\left\|(i \zeta)^{m+n}\left(i \zeta E+\frac{\alpha}{2} E-A\right)^{-m}\left(i \zeta E+\frac{\alpha}{2} E+A\right)^{-n} \tilde{g}(\zeta)\right\|_{L_{2}(R ; H)}^{2} \\
& +\left\|A^{m+n}\left(i \zeta E+\frac{\alpha}{2} E-A\right)^{-m}\left(i \zeta E+\frac{\alpha}{2} E+A\right)^{-n} \tilde{g}(\zeta)\right\|_{L_{2}(R ; H)}^{2} \\
& \leq \sup _{\zeta \in R}\left\|i \zeta^{m+n}\left(i \zeta \mathrm{E}+\frac{\alpha}{2} E-A\right)^{-m}\left(i \zeta \mathrm{E}+\frac{\alpha}{2} E+A\right)^{-n}\right\|_{H \rightarrow H}^{2} \\
& \times\|\tilde{g}(\zeta)\|_{L_{2}(R ; H)}^{2} \\
& +\sup _{\zeta \in R}\left\|A^{m+n}\left(i \zeta \mathrm{E}+\frac{\alpha}{2} E-A\right)^{-m}\left(i \zeta \mathrm{E}+\frac{\alpha}{2} E+A\right)^{-n}\right\|_{H \rightarrow H}^{2} \\
& \times\|\tilde{g}(\zeta)\|_{L_{2}(R ; H)}^{2} .
\end{aligned}
$$


As $\sigma(A)$ is a spectrum of the operator $A$, then:

$$
\begin{aligned}
& \sup _{\zeta \in R}\left\|(i \zeta)^{m+n}\left(i \zeta \mathrm{E}+\frac{\alpha}{2} E-A\right)^{-m}\left(i \zeta \mathrm{E}+\frac{\alpha}{2} E+A\right)^{-n}\right\|_{H \rightarrow H} \\
& \leq \sup _{\zeta \in R \sigma \in \sigma(A)}\left|(i \zeta)^{m+n}\left(i \zeta+\frac{\alpha}{2}-\sigma\right)^{-m}\left(i \zeta+\frac{\alpha}{2}+\sigma\right)^{-n}\right| \\
& =\sup _{\zeta \in R} \frac{|\zeta|^{m+n}}{\left(\zeta^{2}+\left(\sigma+\frac{\alpha}{2}\right)^{2}\right)^{\frac{m+n}{2}}} \leq 1, \\
& \sup _{\zeta \in R}\left\|A^{m+n}\left(i \zeta \mathrm{E}+\frac{\alpha}{2} E-A\right)^{-m}\left(i \zeta \mathrm{E}+\frac{\alpha}{2} E+A\right)^{-n}\right\|_{H \rightarrow H} \\
& \leq \sup _{\zeta \in R \sigma \in \sigma(A)} \sup \left|\sigma^{m+n}\left(i \zeta+\frac{\alpha}{2}-\sigma\right)^{-m}\left(i \zeta+\frac{\alpha}{2}+\sigma\right)^{-n}\right| \\
& =\sup _{\sigma \in \sigma(A)\left(\zeta^{2}+\left(\sigma+\frac{\alpha}{2}\right)^{2}\right)^{\frac{m+n}{2}}} \leq 1 .
\end{aligned}
$$

From (12) and (11) into (10) we obtain:

$$
\|v\|_{W_{2}^{m+n}(R ; H)}^{2} \leq 2\|\tilde{g}(\zeta)\|_{L_{2}(R ; H)}^{2}=2\|g(t)\|_{L_{2}(R ; H)}^{2},
$$

where $\tilde{v}(\zeta)$ and $\tilde{g}(\zeta)$ are the Fourier transforms of the functions $v(t)$ and $g(t)$, respectively. Then $v(t) \in W_{2}^{m+n}(R ; H)$. Similarly we can prove the boundedness of the operator $P_{0, \alpha}$, hence

$$
\left\|P_{0, \alpha} v\right\|_{L_{2}(R ; H)}^{2} \leq \text { const }\|v\|_{W_{2}^{m+n}(R ; H)}^{2} .
$$

Using the Banach theorem on the inverse operator (see [10], [13]), there exists a bounded inverse operator $P_{0, \alpha}^{-1} \quad: \quad L_{2}(R ; H)$ $\rightarrow W_{2}^{m+n}(R ; H)$. Then the theorem is proved.

Remark. The operators of intermediate derivatives:

$$
A^{j} \frac{d^{n+m-j} u}{d t^{n+m-j}}: W_{2, \alpha}^{n+m}(R ; H) \rightarrow L_{2, \alpha}(R ; H), \quad j=\overline{0, m+n},
$$

are continuous.

Now we estimate the norms of intermediate derivative operators participating in the main part of the equation (1) for finding the exact conditions on regular solvability of the investigated equation, expressed only by its operator coefficients.

From Theorem 2, we have that the norms $\left\|P_{0} u\right\|_{L_{2, \alpha}(R ; H)}$ and $\|u\|_{W_{2, \alpha}^{m+n}(R ; H)}$ are equivalent in the space $W_{2, \alpha}^{m+n}(R ; H)$, then we can estimate the norms of intermediate derivatives operators by the norm $\left\|P_{0} u\right\|_{L_{2, \alpha}(R ; H)}($ see [14]). 
Theorem 3. The operator $P_{1}: W_{2, \alpha}^{n+m}(R ; H) \rightarrow L_{2, \alpha}(R ; H)$ is continuous, provided that the operators $A_{j} A^{-j}, j=\overline{0, m+n}$, are bounded in $H$ (see $[2]-[4])$.

Proof. Since $u(t) \in W_{2, \alpha}^{n+m}(R ; H)$ then from the theorem on intermediate derivatives (see [14]), we have

$$
\begin{aligned}
& \left\|P_{1} u\right\|_{L_{2, \alpha}} \leq \sum_{j=0}^{n+m}\left\|A_{j} A^{-j}\right\|_{H \rightarrow H}\left\|A^{j} \frac{d^{n+m-j} u}{d t^{n+m-j}}\right\|_{L_{2, \alpha}} \\
& \leq \text { const }\|u\|_{W_{2, \alpha}^{n+m}(R ; H)} .
\end{aligned}
$$

The theorem is proved.

It follows by Theorem 2 and Theorem 3 that the following lemma is true:

Lemma 4. Consider that the operators $A_{j} A^{-j}, j=\overline{0, n+m}$, are bounded on $H$. Then the operator $P$, in case $A_{j} \neq 0$ acting from the space $W_{2, \alpha}^{n+m}(R ; H)$ to $L_{2, \alpha}$ be bounded (see [15], [16]).

Theorem 5. Let $|\alpha|<2 \sigma_{0}$. Then for any $u(t) \in W_{2, \alpha}^{m+n}(R ; H)$, the following inequalities hold:

$$
\left\|A^{m+n-j} \frac{d^{j} u(t)}{d t^{j}}\right\|_{L_{2, \alpha}} \leq b_{j}\left\|P_{0} u\right\|_{L_{2, \alpha}},
$$

where

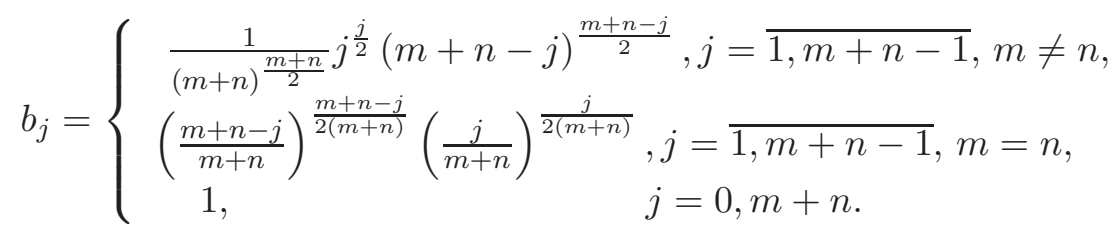

Proof. Apply the Fourier transformation on equation (6) (see [1]-[3]), we get

$$
\begin{aligned}
& \left\|A^{m+n-j}(i \zeta)^{j}\left(i \zeta \mathrm{E}+\frac{\alpha}{2} E-A\right)^{-m}\left(i \zeta \mathrm{E}+\frac{\alpha}{2} E+A\right)^{-n} \tilde{g}(\zeta)\right\|_{L_{2}(R ; H)} \\
& \leq \sup _{\zeta \in R}\left\|A^{m+n-j}(i \zeta)^{j}\left(i \zeta \mathrm{E}+\frac{\alpha}{2} E-A\right)^{-m}\left(i \zeta \mathrm{E}+\frac{\alpha}{2} E+A\right)^{-n}\right\|_{H \rightarrow H} \\
& \times\|\tilde{g}(\zeta)\|_{L_{2}(R ; H)} .
\end{aligned}
$$


For $\zeta \in R$, we estimate the following norms:

$$
\begin{aligned}
& \left\|A^{m+n-j}(i \zeta)^{j}\left(i \zeta \mathrm{E}+\frac{\alpha}{2} E-A\right)^{-m}\left(i \zeta \mathrm{E}+\frac{\alpha}{2} E+A\right)^{-n}\right\|_{H \rightarrow H} \\
& \leq \sup _{\sigma \in \sigma(A)}\left|\sigma^{m+n-j}(i \zeta)^{j}\left(i \zeta+\frac{\alpha}{2}-\sigma\right)^{-m}\left(i \zeta+\frac{\alpha}{2}+\sigma\right)^{-n}\right| \\
& =\sup _{\sigma \in \sigma(A)}\left|\sigma^{-j}(i \zeta)^{j}\left(i \frac{\zeta}{\sigma}-\left(-\frac{\alpha}{2 \sigma}+1\right)\right)^{-m}\left(i \frac{\zeta}{\sigma}+\left(\frac{\alpha}{2 \sigma}+1\right)\right)^{-n}\right|
\end{aligned}
$$

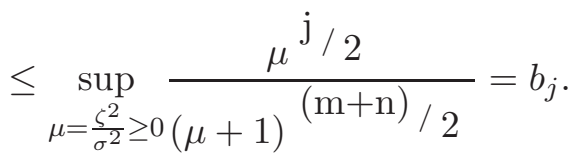

Finally, from (15) we have

$$
\left\|A^{m+n}(i \zeta)^{j}(i \mathrm{E}-A)^{-m}(i \zeta \mathrm{E}+A)^{-n} \tilde{f(\zeta)}\right\|_{L_{2, \alpha}} \leq b_{j}\|\tilde{f(\zeta)}\|_{L_{2, \alpha}} .
$$

Now, we introduce the following specific cases at certain values of $m$ and $n$ :

Case (i) $m=3, n=0$, then we have an initial-boundary value problem of a third order operator-differential equation with multiple characteristics with

$$
b_{1}=b_{2}=\frac{2}{3 \sqrt{3}}(\text { see }[23]) \text {. }
$$

Case (ii) $m=0, n=3$, then we have an initial-boundary value problem of a fourth order operator-differential equation with multiple characteristics with

$$
b_{1}=b_{2}=\frac{2}{3 \sqrt{3}}(\text { see }[17]) \text {. }
$$

Case (iii) $m=3, n=1$, then we have an initial-boundary value problem of a fourth order operator-differential equation with multiple characteristics with

$$
b_{1}=b_{3}=\frac{3 \sqrt{3}}{16}, b_{2}=\frac{1}{4} \text {. }
$$

Case (iv) $m=1, n=3$, then we have an initial-boundary value problem of a fourth order operator-differential equation with complicated characteristics with

$$
b_{1}=b_{3}=\frac{3 \sqrt{3}}{16}, b_{2}=\frac{1}{4}(\text { see }[7]) .
$$

Now in $H_{2(m+n)}$ we consider the following pencil operator

$$
Q_{j}(\lambda, \beta, A)=\left\{\begin{array}{c}
\left((i \lambda)^{2} E+A^{2}\right)^{m+n}-\beta(i \lambda)^{2 j} A^{2(m+n)-2 j}, m \neq n, \\
\lambda^{2(m+n)} E+A^{2(m+n)}-\beta(i \lambda)^{2 j} A^{2(m+n)-2 j}, m=n,
\end{array}\right.
$$


$\beta$ is a real parameter. From (18) we have

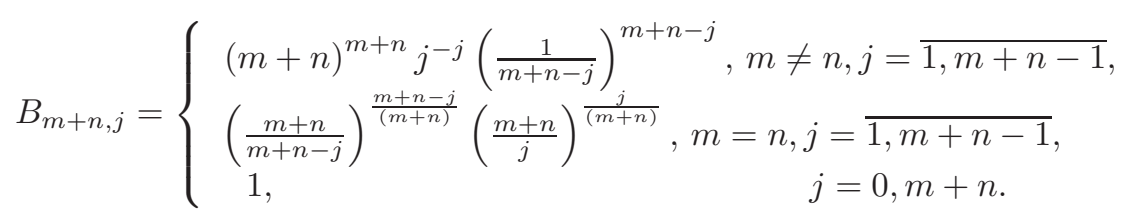

Theorem 6. For $\beta \in\left[0, B_{m+n, j}\right), j=\overline{0, m+n}$, the operator pencils $Q_{j}(\lambda, \beta, A)$ can be represented in the form

$$
Q_{j}(\lambda, \beta, A)=Q_{j}^{-}(\lambda, \beta, A) Q_{j}^{+}(-\lambda, \beta, A), j=\overline{0, m+n},
$$

where

$$
\begin{aligned}
Q_{j}^{-}(\lambda, \beta, A) & =\sum_{i=0}^{m+n} \alpha_{i, j} \lambda^{i} A^{m+n-i}, \\
Q_{j}^{+}(-\lambda, \beta, A) & =\sum_{i=0}^{m+n} \alpha_{i, j}(-\lambda)^{i} A^{m+n-i},
\end{aligned}
$$

and satisfy the following system of equations:

$$
Q_{j}(\lambda, \beta, A)=\left(\sum_{i=0}^{m+n} \alpha_{i, j} \lambda^{i} A^{m+n-i}\right)\left(\sum_{i=0}^{m+n} \alpha_{i, j}(-\lambda)^{i} A^{m+n-i}\right) .
$$

Proof. For $\beta \in\left[0, B_{m+n, j}\right), j=\overline{0, m+n}$, the polynomial

$$
Q_{j}(\lambda, \beta)=\left\{\begin{array}{c}
\left((i \lambda)^{2}+1\right)^{m+n}-\beta(i \lambda)^{2 j}, m \neq n, \\
\lambda^{2(m+n)}+1-\beta(i \lambda)^{2 j}, m=n,
\end{array}\right.
$$

has not purely imaginary roots, its roots are simple and symmetrically situated relatively to the real axis and the origin. So it can be represented in the form

$$
\begin{gathered}
Q_{j}(\lambda, \beta)=Q_{j}^{-}(\lambda, \beta) Q_{j}^{+}(-\lambda, \beta), \\
Q_{j}^{-}(\lambda, \beta)=\prod_{i=1}^{m+n}\left(\lambda-\omega_{i, j}(\beta)\right),
\end{gathered}
$$

where $\omega_{i, j}(\beta)$ are the roots of $Q_{j}^{-}(\lambda, \beta), \operatorname{Re} \omega_{i, j}(\beta)<0$, hence

$$
Q_{j}^{-}(\lambda, \beta)=\sum_{i=1}^{m+n} \alpha_{i, j}(\beta) \lambda^{i}, \alpha_{i, j}>0 \text { are real coefficients. }
$$


Let $E_{\sigma}$ be the spectral decomposition of $A$.

For the case $m \neq n$ :

$$
\begin{aligned}
& Q_{j}(\lambda, \beta, A)=\left((i \lambda)^{2} E+A^{2}\right)^{m+n}-\beta(i \lambda)^{2 j} A^{2(m+n)-2 j} \\
& =\int_{\sigma^{0}}^{\infty}\left((i \lambda)^{2}+\sigma^{2}\right)^{m+n}-\beta(i \lambda)^{2 j} \sigma^{2(m+n)-2 j} d E_{\sigma} \\
& =\int_{\sigma^{0}}^{\infty} \sigma^{2(m+n)}\left[\left(\frac{(i \lambda)^{2}}{\sigma^{2}}+1\right)^{m+n}-\beta \frac{(i \lambda)^{2 j}}{\sigma^{2 j}}\right] d E_{\sigma} \\
& =\int_{\sigma^{0}}^{\infty} \sigma^{2(m+n)} Q_{j}\left(\frac{\lambda}{\sigma}, \beta\right) d E_{\sigma} \\
& =\int_{\sigma^{0}}^{\infty} \sigma^{2(m+n)} Q_{j}^{-}\left(\frac{\lambda}{\sigma}, \beta\right) Q_{j}^{+}\left(-\frac{\lambda}{\sigma}, \beta\right) d E_{\sigma} \\
& =\int_{\sigma^{0}}^{\infty} \sigma^{(m+n)} Q_{j}^{-}\left(\frac{\lambda}{\sigma}, \beta\right) d E_{\sigma} \int_{\sigma^{0}}^{\infty} \sigma^{(m+n)} Q_{j}^{+}\left(-\frac{\lambda}{\sigma}, \beta\right) d E_{\sigma} \\
& =\left(\sum_{i=0}^{m+n} \alpha_{i, j}(\beta) \lambda^{i} A^{m+n-i}\right)\left(\sum_{i=0}^{m+n} \alpha_{i, j}(\beta)(-\lambda)^{i} A^{m+n-i}\right) .
\end{aligned}
$$

By taking

$$
Q_{j}^{-}(\lambda, \beta, A)=\sum_{i=o}^{m+n} \alpha_{i, j}(\beta) \lambda^{i} A^{m+n-i}
$$

we get

$$
Q_{j}(\lambda, \beta, A)=Q_{j}^{-}(\lambda, \beta, A) Q_{j}^{+}(-\lambda, \beta, A) .
$$

Similarly for the case $m=n$.

For example,

(i) If $m=1, n=3$ satisfy the following system of equations:

(1) for $j=0$

$$
\left\{\begin{array}{l}
\alpha_{0,0}(\beta)=\sqrt{1-\beta} \\
2 \alpha_{2,0}(\beta)-\alpha_{3,0}^{2}(\beta)+4=0 \\
2 \alpha_{0,0}(\beta) \alpha_{4,0}(\beta)+\alpha_{2,0}^{2}(\beta)-2 \alpha_{1,0}(\beta) \alpha_{3,0}(\beta)-6=0 \\
2 \alpha_{0,0}(\beta) \alpha_{2,0}(\beta)-\alpha_{1,0}^{2}(\beta)+4=0
\end{array}\right.
$$

(2) for $j=1$

$$
\left\{\begin{array}{l}
2 \alpha_{2,1}(\beta)-\alpha_{3,1}^{2}(\beta)+4=0 \\
2 \alpha_{0,1}(\beta) \alpha_{4,1}(\beta)+\alpha_{2,1}^{2}(\beta)-2 \alpha_{1,1}(\beta) \alpha_{3,1}(\beta)-6=0 \\
2 \alpha_{0,1}(\beta) \alpha_{2,1}(\beta)-\alpha_{1,1}^{2}(\beta)+4=\beta
\end{array}\right.
$$


(3) for $j=2$

$$
\left\{\begin{array}{l}
2 \alpha_{2,2}(\beta)-\alpha_{3,2}^{2}(\beta)+4=0 \\
2 \alpha_{0,2}(\beta) \alpha_{4,2}(\beta)+\alpha_{2,2}^{2}(\beta)-2 \alpha_{1,2}(\beta) \alpha_{3,2}(\beta)-6+\beta=0 \\
2 \alpha_{0,2}(\beta) \alpha_{2,2}(\beta)-\alpha_{1,2}^{2}(\beta)+4=0
\end{array}\right.
$$

(4) for $j=3$

$$
\left\{\begin{array}{l}
2 \alpha_{2,3}(\beta)-\alpha_{3,3}^{2}(\beta)+4-\beta=0 \\
2 \alpha_{0,3}(\beta) \alpha_{4,3}(\beta)+\alpha_{2,3}^{2}(\beta)-2 \alpha_{1,3}(\beta) \alpha_{3,3}(\beta)-6=0 \\
2 \alpha_{0,3}(\beta) \alpha_{2,3}(\beta)-\alpha_{1,3}^{2}(\beta)+4=0
\end{array}\right.
$$

(5) for $j=4$

$$
\left\{\begin{array}{l}
\alpha_{4,4}(\beta)=\sqrt{1+\beta} \\
2 \alpha_{2,4}(\beta)-\alpha_{3,4}^{2}(\beta)+4=0 \\
2 \alpha_{0,4}(\beta) \alpha_{4,4}(\beta)+\alpha_{2,4}^{2}(\beta)-2 \alpha_{1,4}(\beta) \alpha_{3,4}(\beta)-6=0 \\
2 \alpha_{0,4}(\beta) \alpha_{2,4}(\beta)-\alpha_{1,4}^{2}(\beta)+4=0
\end{array}\right.
$$

(ii) If $m=2, n=2$ satisfy the following system of equations:

(1) for $j=0$

$$
\left\{\begin{array}{l}
\alpha_{0,0}(\beta)=\sqrt{1-\beta} \\
\alpha_{1,0}^{2}(\beta)-2 \alpha_{0,0}(\beta) \alpha_{2,0}(\beta)=0 \\
\alpha_{2,0}^{2}(\beta)-2 \alpha_{1,0}(\beta) \alpha_{3,0}(\beta)+2 \alpha_{0,0}(\beta)=0 \\
\alpha_{3,0}^{2}(\beta)-2 \alpha_{2,0}(\beta)=0
\end{array}\right.
$$

(2) for $j=1$

$$
\left\{\begin{array}{l}
\alpha_{3,1}^{2}(\beta)^{2}-2 \alpha_{2,1}(\beta)=0 \\
\alpha_{1,1}^{2}(\beta)-2 \alpha_{2,1}(\beta)+\beta=0 \\
\alpha_{2,1}^{2}(\beta)-2 \alpha_{1,1}(\beta) \alpha_{3,1}(\beta)+2=0
\end{array}\right.
$$

(3) for $j=2$

$$
\left\{\begin{array}{l}
\alpha_{1,2}^{2}(\beta)-2 \alpha_{2,2}(\beta)=0 \\
\alpha_{3,2}^{2}(\beta)-2 \alpha_{2,2}(\beta)=0 \\
\alpha_{2,2}^{2}(\beta)-2 \alpha_{1,2}(\beta) \alpha_{3,2}(\beta)+2=-\beta
\end{array}\right.
$$

(4) for $j=3$

$$
\left\{\begin{array}{l}
\alpha_{3,3}(\beta)-2 \alpha_{2,3}(\beta)=-\beta \\
\alpha_{1,3}^{2}(\beta)-2 \alpha_{2,3}(\beta)=0 \\
\alpha_{2,3}^{2}(\beta)-2 \alpha_{1,3}(\beta) \alpha_{3,3}(\beta)+2=0
\end{array}\right.
$$


(5) for $j=4$

$$
\left\{\begin{array}{l}
\alpha_{4,4}(\beta)=\sqrt{1-\beta} \\
\alpha_{1,4}^{2}(\beta)-2 \alpha_{0,4}(\beta) \alpha_{2,4}(\beta)=0 \\
\alpha_{2,4}^{2}(\beta)-2 \alpha_{1,4}(\beta) \alpha_{3,4}(\beta)+2 \alpha_{4,4}(\beta)=0 \\
\alpha_{3,4}^{2}(\beta)-2 \alpha_{2,4}(\beta) \alpha_{4,4}(\beta)=0
\end{array}\right.
$$

Lemma 7. Let $\beta \in\left[0, B_{m+n, j}\right)$, then for any $u \in W_{2, \alpha}^{m+n}(R ; H)$

$$
\begin{aligned}
& \left\|Q_{j}^{-}\left(\frac{d}{d t} ; \beta ; A\right) u\right\|_{L_{2}(R ; H)}^{2} \\
& =\left\|P_{0} u\right\|_{L_{2, \alpha}}^{2, \alpha}-\beta\left\|A^{m+n-j} \frac{d^{j}}{d t^{j}} u\right\|_{L_{2, \alpha}}^{2}, j=\overline{0, m+n} .
\end{aligned}
$$

From Theorem 5 and the theorem of intermediate derivatives (see [18]) the norms $\|u\|_{W_{2, \alpha}^{m+n}(R ; H)}$ and $\left\|P_{0} u\right\|_{L_{2}(R ; H)}$ are equivalent in the space $W_{2, \alpha}^{m+n}(R ; H)$. Therefore, the numbers

$$
N_{j}=\sup _{0 \neq u \in W_{2, \alpha}^{m+n}(R ; H)} \frac{\left\|A^{m+n-j} \frac{d^{j}}{d t^{j}} u\right\|_{L_{2, \alpha}}}{\left\|P_{0} u\right\|_{L_{2, \alpha}}}, j=\overline{0, m+n},
$$

are finite numbers. To find exact values of these numbers, we provide the following lemma:

Theorem 8. The numbers $N_{j}$ are determined as follows:

$$
N_{j}=b_{j}, j=\overline{0, m+n} .
$$

Proof. As (22) goes to the limit as $\beta \rightarrow B_{m+n, j}$, it is clear that for any vector function $u(t) \in W_{2, \alpha}^{m+n}(R ; H)$ :

$$
\left\|P_{0} u\right\|_{L_{2, \alpha}}^{2} \geq B_{m+n, j}\left\|A^{m+n-j} \frac{d^{j}}{d t^{j}} u\right\|_{L_{2, \alpha}}^{2}, j=\overline{0, m+n} .
$$

So, $N_{j} \leq B_{m+n, j}, j=\overline{0, m+n}$. Moreover, we must show that $N_{j}=B_{m+n, j}, j=$ $\overline{0, m+n}$ is also hold. To do this, it is sufficient for any $\delta>0$, there exist a vector function $u_{\delta}(t) \in W_{2, \alpha}^{m+n}(R ; H)$ such that the functional

$$
\chi\left(u_{\delta}(t)\right) \equiv\left\|P_{0} u_{\delta}\right\|_{L_{2, \alpha}}^{2}-\left(\delta+B_{m+n, j}\right)\left\|A^{m+n-j} \frac{d^{j}}{d t^{j}} u_{\delta}\right\|_{L_{2, \alpha}}^{2}<0 .
$$


Let the vector $\nu \in D\left(A^{m+n}\right)$ such that $\|\nu\|=1, r(t) \in W_{2, \alpha}^{m+n}(R ; H)$ be scalar function. Using Parseval's equality, we obtain

$$
\begin{aligned}
\chi(r(t) \nu) & =\left\|P_{0}(r(t) \nu)\right\|_{L_{2, \alpha}}^{2}-\left(\delta+B_{m+n, j}\right)\left\|A^{m+n-j} \frac{d^{j} r(t)}{d t^{j}} \nu\right\|_{L_{2, \alpha}}^{2} \\
& =\int_{-\infty}^{+\infty}\left(\left(P_{0}(-i \zeta ; A) \nu, P_{0}(-i \zeta ; A) \nu\right)|\tilde{r}(\zeta)|^{2}\right. \\
& \left.-\zeta^{2 j}\left(\delta+B_{m+n, j}\right)\left(A^{m+n-j} \nu, A^{m+n-j} \nu\right)|\tilde{r}(\zeta)|^{2}\right) d \zeta \\
& =\int_{-\infty}^{+\infty}\left(P_{0}(-i \zeta ; A) P_{0}(-i \zeta ; A) \nu\right. \\
& \left.\left.-\zeta^{2 j}\left(\delta+B_{m+n, j}\right) A^{2(m+n-j)} \nu, \nu\right)|\tilde{r}(\zeta)|^{2}\right) d \zeta \\
& =\int_{-\infty}^{+\infty}\left(P_{j}\left(i \zeta ; \delta+B_{m+n, j} ; A\right) \nu, \nu\right)|\tilde{r}(\zeta)|^{2} d \zeta,
\end{aligned}
$$

where $\tilde{r}(\zeta)$ is the Fourier transform of $r(t)$. Then it is necessary to show that $\left(P_{j}\left(i \zeta ; \delta+B_{m+n, j} ; A\right) \nu, \nu\right)$ for a given vector $\nu$ has negative values in some interval $\left(\epsilon^{-}, \epsilon^{+}\right)$. For $\sigma_{0}>0$ is an eigenvalue of $A$, and $\zeta$ is its corresponding eigenvector, then

$$
\left(P_{j}\left(i \zeta ; \delta+B_{m+n, j} ; A\right) \nu, \nu\right)=\left(P_{j}\left(i \zeta ; \delta+B_{m+n, j} ; \sigma_{0}\right) \nu, \nu\right) .
$$

From the properties of the polynomial $P_{j}\left(i \zeta ; \beta ; \sigma_{0}\right)$, it is negative for $\beta=\delta+$ $B_{m+n, j}$ for sufficiently small $\delta>0$. If $\sigma_{0} \in \sigma(A)$ is not an eigenvalue, then $\sigma_{0}$ is close to an eigenvalue, i.e. there exist a vector $\nu_{\delta}$ such that $\left\|\nu_{\delta}\right\|=1$ and

$$
\begin{gathered}
\left(P_{j}\left(i \zeta ; \delta+B_{m+n, j} ; A\right) \nu_{\delta}, \nu_{\delta}\right)=\left(P_{j}\left(i \zeta ; \delta+B_{m+n, j} ; \sigma_{0}\right) \nu_{\delta}, \nu_{\delta}\right)+0(\delta) \\
\text { as } \delta \rightarrow 0 .
\end{gathered}
$$

In which, the smallest value of $\left.\left(P_{j}\left(i \zeta ; \delta+B_{m+n, j} ; A\right) \nu, \nu\right)\right)$ is negative for sufficiently small $\delta$ and some $\nu_{\delta}$. Then there exist an interval $\left(\epsilon^{-}, \epsilon^{+}\right)$such that

$$
\left.\left(P_{j}\left(i \zeta ; \delta+B_{m+n, j} ; A\right) \nu, \nu\right)\right)<\delta, \quad \zeta \in\left(\epsilon^{-}, \epsilon^{+}\right) .
$$

Further, we consider the $m+n$ times differentiable function $\tilde{r}(\zeta), \zeta \in\left(\epsilon^{-}, \epsilon^{+}\right)$, then from the negativity of $\left.\left(P_{j}\left(i \zeta ; \delta+B_{m+n, j} ; A\right) \nu_{\delta}, \nu_{\delta}\right)\right)$ in the interval $\left(\epsilon^{-}, \epsilon^{+}\right)$ and from (25), we obtain

$$
\chi\left(r(t) \nu_{\delta}\right)=\int_{\epsilon^{-}}^{\epsilon^{+}}\left(P_{j}\left(i \zeta ; \delta+B_{m+n, j} ; A\right) \nu_{\delta}, \nu_{\delta}\right)|\tilde{r}(\zeta)|^{2} d \zeta<0 .
$$

Consequently, $N_{j}=b_{j}, j=\overline{0, m+n}$. 
Theorem 9. Let $A=A^{*} \geq \sigma_{0} E\left(\sigma_{0}>0\right),|\alpha|<2 \sigma_{0}$, the operators $A_{j} A^{-j}, j=\overline{0, m+n}$ are bounded in $H$ and holds the inequality

$$
\sum_{j=0}^{m+n} b_{j}\left\|A_{m+n-j} A^{-(m+n-j)}\right\|_{H \rightarrow H}<1,
$$

in which the numbers $b_{j}, j=\overline{0, m+n}$, are determined in Theorem 5 , then the equation (1) is regularly solvable.

Proof. From Theorem 2, the operator $P_{0}$ has a bounded inverse operator $P_{0}^{-1}$ acting from $L_{2, \alpha}(R ; H)$ to $W_{2, \alpha}^{m+n}(R ; H)$. Then equation (1) can be written as

$$
\left(E+P_{1} P_{0}^{-1}\right) z(t)=f(t)
$$

where $P_{0} u(t)=z(t)$. To prove the existence of a solution, we must show that the norm

$$
\left\|p_{1} p_{0}^{-1}\right\|_{L_{2, \alpha}(R ; H) \rightarrow L_{2, \alpha}(R ; H)}<1
$$

By Theorem 2, we have

$$
\begin{aligned}
& \left\|P_{1} P_{0}^{-1} z\right\|_{L_{2, \alpha}}=\left\|P_{1} u\right\|_{L_{2, \alpha}} \leq \sum_{j=0}^{m+n}\left\|A_{j} \frac{d^{m+n-j} u}{d t^{m+n-j}}\right\|_{L_{2, \alpha}} \\
& \leq \sum_{j=0}^{m+n}\left\|A_{j} A^{-j}\right\|_{H \rightarrow H}\left\|A^{j} \frac{d^{m+n-j} u}{d t^{m+n-j}}\right\|_{L_{2, \alpha}} \\
& \leq \sum_{j=0}^{m+n} b_{j}\left\|A_{j} A^{-j}\right\|_{H \rightarrow H}\left\|P_{0} u\right\|_{L_{2, \alpha}} \\
& =\sum_{j=0}^{m+n} b_{j}\left\|A_{j} A^{-j}\right\|_{H \rightarrow H}\|z\|_{L_{2, \alpha}} .
\end{aligned}
$$

Consequently,

$$
\left\|P_{1} P_{0}^{-1}\right\|_{L_{2, \alpha} \rightarrow L_{2, \alpha}} \leq \sum_{i=0}^{m+n} b_{j}\left\|A_{j} A^{-j}\right\|_{H \rightarrow H}<1 .
$$

Providing that the operator $E+P_{1} P_{0}^{-1}$ is invertible in $L_{2}(R ; H)$, hence $u(t)$ can be determined by $u(t)=P_{0}^{-1}\left(E+P_{1} P_{0}^{-1}\right)^{-1} f(t)$. Moreover,

$$
\begin{aligned}
& \|u\|_{W_{2, \alpha}^{m+n}} \leq\left\|p_{0}^{-1}\right\|_{L_{2, \alpha} \rightarrow W_{2, \alpha}^{m+n}(R ; H)}\|f\|_{L_{2, \alpha}(R ; H)} \\
& \times\left\|\left(\left(E+P_{1} P_{0}^{-1}\right)\right)^{-1}\right\|_{L_{2}(R ; H) \rightarrow L_{2, \alpha}} \| \text { const }\|f\|_{L_{2, \alpha}} .
\end{aligned}
$$

The theorem is proved.

Consider a polynomial operator pencil (see [8]) of a $m+n$ order in $H$ is

$$
P(\lambda)=\prod_{k=1}^{m}\left(\lambda+\mu_{k} A\right) \prod_{k=1}^{n}\left(\lambda+\mu_{k} A\right)+\sum_{j=1}^{m+n-1} A_{m+n-j} \lambda^{(j)},
$$




$$
\begin{gathered}
P_{0}(\lambda)=\prod_{k=1}^{m}\left(\lambda+\mu_{k} A\right) \prod_{k=1}^{n}\left(\lambda+\mu_{k} A\right), \\
P_{1}(\lambda)=\sum_{j=1}^{m+n-1} A_{m+n-j} \lambda^{(j)} .
\end{gathered}
$$

According to Theorem 3, it suffices to establish the following theorem (see [5], $[21],[20])$.

Theorem 10. Suppose that the operators $A_{j} A^{-j}$ are bounded operators in $H, j=\overline{0, m+n-1}$ and the inequality

$$
\sum_{j=0}^{m+n-1} b_{j}\left\|A_{j} A^{-j}\right\|_{H \rightarrow H}<1,
$$

holds true, where $b_{j}, j=\overline{0, m+n}$ are calculated in Theorem 5 , then the resolvent of the pencil (28) exists on the imagery axis and the inequalities

$$
\begin{gathered}
\sum_{r=0}^{m+n-1}\left\|\lambda^{m+n-r} A^{r} P^{-1}(\lambda)\right\| \leq \text { const }, \\
\left\|A^{\alpha} P^{-1}(\lambda)\right\| \leq \text { const }|\lambda|^{\alpha-(m+n)}, \quad 0<\alpha<m+n, \lambda \neq 0
\end{gathered}
$$

hold.

Theorem 11. From Theorem 10, for sufficiently small $\phi$ (greater than zero) on the sectors

$$
\begin{gathered}
\Gamma_{\frac{\pi}{2} \pm \phi}=\left\{\lambda: \lambda=r e^{i\left(\frac{\pi}{2} \pm \phi\right)}, r>0\right\}, \\
\Gamma_{\frac{-\pi}{2} \pm \phi}=\left\{\lambda: \lambda=r e^{-i\left(\frac{\pi}{2} \pm \phi\right)}, r>0\right\},
\end{gathered}
$$

the operator pencil (28) is invertible and the estimates of the form (29) and (30) hold.

\section{Example}

As a result of the solvability of the differential equation (1), we introduce the following problem of partial differential equation as an applied example on the strip $R \times[0 ; \pi]$ :

$$
\left(\frac{\partial}{\partial t}+\frac{\partial^{2}}{\partial x^{2}}\right)^{m}\left(\frac{\partial}{\partial t}-\frac{\partial^{2}}{\partial x^{2}}\right)^{n} u(t, x)
$$




$$
\begin{gathered}
+\sum_{j=0}^{n+m} r_{n+m-j}(x) \frac{\partial^{2(n+m)-j} u(t, x)}{\partial t^{j} \partial x^{2(n+m-j)}}=f(t, x), \\
\frac{\partial^{2 k} u(t, 0)}{\partial x^{2 k}}=\frac{\partial^{2 k} u(t, \pi)}{\partial x^{2 k}}=0, k=\overline{0, n+m},
\end{gathered}
$$

where $r_{n+m-j}(x), j=\overline{0, n+m}$ are bounded functions on $[0, \pi], f(t, x) \in$ $L_{2}\left(R ; L_{2}[0 ; \pi]\right)$. We note that problem $(31),(32)$ is a special case and can be reduced to the operator-differential equation (1) in which:

$$
A_{j}=r_{n+m-j}(x) \frac{\partial^{2(n+m-j)}}{\partial x^{2(n+m-j)}}, \quad j=\overline{0, n+m} .
$$

The operator $A$ is defined on $H=L_{2}[0, \pi]$ by $A u=-\frac{d^{2} u}{d x^{2}}$, and the conditions $\left.u\right|_{x=0}=\left.u\right|_{x=\pi}=0$. Applying Theorem 10, where

$$
\sum_{j=0}^{m+n} b_{j} \sup _{x \in[0, \pi]}\left|r_{j}(x)\right|<1
$$

then problem (31)-(32) has a unique solution

$$
u(x, t) \in W_{t, x, 2}^{n+m, 2(n+m)}\left(R ; L_{2}[0 ; \pi]\right) .
$$

\section{Conclusion}

In a weighted Sobolev space for all $t \in R$, we calculated the exact conditions of regular solvability of equation (1), expressed only by its operator coefficients. We deduced the relationship between the exponent of the weight $\left(e^{\frac{-\alpha t}{2}}\right)$ and the lower bound $\left(\sigma_{0}\right)$ of the spectrum of the operator of the main part of the equation. We estimated the norms of intermediate derivative operators participating in the principle part of the given equation. In the perturbed part of equation (1), the norms of the linear operators $A_{j}(t), j=\overline{0, m+n}$, were estimated. An alternative method on the regular solvability of operator pencil was investigated. As an applied result, we formulated a problem of $2(m+n)$ order partial differential equations.

\section{References}


[1] A.B.I. Ahmed, M.A. Labeeb, Solvability of a class of operator-differential equations of third order with complicated characteristic on the whole real axis, Open Access Library J., 5 (2018), 1-5.

[2] A.B.I. Ahmed, Solvability of a non-homogeneous boundary value problem for a class of higher-order operator-differential equations in a weighted Space, Technology Reports of Kansai Univ., 62 (2020), 5551-5561.

[3] A.B.I. Ahmed, Solvability of initial-boundary value problem for parabolic $n$ th-order operator-differential equations in Hilbert space, Technology Reports of Kansai Univ., 62 (2020), 373-381.

[4] N. Faried, L. Rashed, A.B.I. Ahmed, M.A. Labeeb, Solvability of initialboundary value problem of a multiple characteristic fifth-order operatordifferential equation, J. Egypt. Math. Soc., 27 (2019), 1-7.

[5] N. Faried, A.B.I. Ahmed, M.A. Labeeb, Sufficient conditions for regular solvability of an arbitrary order operator-differential equation with initialboundary conditions, Adv. in Difference Equations, 2020, No 104 (2020), 1-14.

[6] E.G. Gamidov, On a boundary value problem for second order operator differential equations in space of smooth vector functions, Trans. of NAS of Azerbaijan, XXXIII (2013), 72-84.

[7] A.A. Gasymov, On solvability of a class of complicated characteristic operator differential equations of fourth order, Trans. of NAS of Azerbaijan, 1, No 28 (2007), 49-54.

[8] M.G. Gasymov, The multiple completeness of part of the eigen- and associated vectors of polynomial operator bundles, Izv. Akad. Nauk Arm. SSR, Ser. Matem, 6, No 2-3 (1971), 131-147.

[9] V.I. Gorbachuk, M.L. Gorbachuk, Boundary Value Problems for Differential-Operator Equations, Kiev, Naukova Dumka (1984) (In Russian).

[10] E. Hille, R. Phillips, Functional Analysis and Semi-Group, Revis. Ed., Amer. Math. Soc. Colloq. Publ., 31, Amer. Math. Soc., Providence, R.I. (1957); Russian transl., IL, Moscow (1962).

[11] R.Z. Humbataliev, On regular solvability of boundary value problems in weight space, Intern. J. of Math. Analysis, 1 (2007), 1209-1216. 
[12] V. Jabrayilova, On double completeness of part of root vectors of a class of ploynomial operator bundles of the fourth order, Trans. of NAS of Azerbaijan, 7, No 25 (2005), 55-60.

[13] S.G. Krein, Linear Equations in Banach Spaces, Birkhäuser, Boston (1982).

[14] E. Kreyszig, Introductory Functional Analysis with Applications, USA (1989).

[15] N.V. Krylov, Lectures on Elliptic and Parabolic Equations in Sobolev Spaces, American Mathematical Society, 96 (2008).

[16] M.A. Labeeb, A.B.I. Ahmed, L. Rashed, Conditional solvability of the boundary value problem of a self-adjoint operator-differential equations in a Sobolev-type space, International Journal of Applied Mathematics, 32, No 3 (2019), 469-478; doi:10.12732/ijam.v32i3.8.

[17] F.S. Lachinova, Solvability of a class of parabolic operator-differential equations of third order, Proc. of IMM of NAS of Azerbaijan, XXXIX (2013), 77-86.

[18] J.L. Lions, E. Majenes, Non-Homogeneous Boundary Value Problems and Applications, Springer-Verlag, Berlin-Heidelberg-New York (1972).

[19] S.S. Mirzoev, On solvability of boundary value problems for operator differential equations of the second order in spaces with weight, In: Linear Operators and Their Applications, ASU, Baku (1989), 46-49 (In Russian).

[20] S.S. Mirzoev, F.A. Gulieva, On the completeness of elementary solutions of a class of second order differential equations with operator coefficients, Mathematical Notes, 86, No 5 (2009), 747-750.

[21] S.S. Mirzoev, M.D. Karaaslan, R.Z. Gumbataliev, To the theory of operator differential equations of the second order, Doklady Mathematics, $8 \mathbf{8}$ (2013), 741-743.

[22] V.N. Pilipchuk, On essentially nonlinear dynamics of arches and rings, $J$. of Appl. Math. and Mech., 46, No 3 (1982), 360-364.

[23] M.A. Soylemez, Solvability conditions for one inverse parabolic operator differential equation in sobolev-type space, J. of Qafqaz Univ.-Mathematics and Computer Sci., 4, No 1 (2016), 95-100. 
[24] G.A. Teters, Complex Loading and Stability of the Covers from Polymeric Materials, Latvia, Riga, Zinatne Press (In Russian) (1969).

[25] S. Yakubov, Y. Yakubov, Differential Operator Equations: Ordinary and Partial Differential Equations, Ser. Pure and Applied Mathematics, Chapman and Hall/CRC, USA (1999). 
